\title{
Aquaporin-4 and myelin oligodendrocyte glycoprotein antibodies in immune mediated optic neuritis at long-term follow-up
}

\author{
A. Petzold ${ }^{a, b, c^{*}}$ M. Woodhall ${ }^{d}$ Z. Khaleeli ${ }^{b}$ W.O. Tobin e S. Pittock e,f,g \\ B.G. Weinshenker ${ }^{f}$ A. Vincent ${ }^{d}$ P. Waters ${ }^{d}$ G.T. Plant ${ }^{b, c, h}$
}

\begin{abstract}
a Department of Neuroinflammation, UCL Institute of Neurology, Queen Square, London WC1N 3BG, UK b National Hospital for Neurology and Neurosurgery and Moorfields Eye Hospital, London, UK

cNeuro-ophthalmology Expertise Centre, Departments of Neurology and Ophthalmology, Amsterdam UMC, Amsterdam, NL. d Nuffield Department of Clinical Neurosciences, University of Oxford, Oxford, UK. e Department of Neurology, Mayo Clinic College of Medicine, Rochester, MN 55905, USA f Department of Laboratory Medicine and Pathology, Mayo Clinic College of Medicine, Rochester, MN 55905, USA g Department of Immunology, Mayo Clinic College of Medicine, Rochester, MN 55905, USA

h Medical Eye Unit, St. Thomas' Hospital, Lambeth Palace Road London, SE1 7EH, UK

* Corresponding author: Axel Petzold, UCL ION, Queen Square, London, WC1N 3BG, UK; Email: a.petzold@ucl.ac.uk
\end{abstract}

Word count abstract: 236

Word count main text: 2448

References: 45 


\section{Abstract}

Objectives: To re-evaluate serum samples from our 2007 cohort of patients with single episode isolated ON (SION), recurrent isolated ON (RION), chronic relapsing inflammatory optic neuropathy (CRION), multiple sclerosis-associated ON (MSON) and neuro-myelitis optica (NMO).

Methods: We re-screened 103/114 patients with available serum on live cell based assays for AQP4-M23-IgG and MOG- $\alpha$ 1-lgG. Further testing included oligoclonal bands, serum levels of glial fibrillary acidic and neurofilament proteins and S100B. We show the impact of updated serology on these patients.

Results: Reanalysis of our the original cohort revealed that AQP4-IgG seropositivity increased from $56 \%$ to $75 \%$ for NMO, $5 \%$ to $22 \%$ for CRION, $6 \%$ to $7 \%$ for RION, $0 \%$ to $7 \%$ for $\mathrm{MSON}$ and $5 \%$ to $6 \%$ for SION. MOG-IgG1 was identified in $25 \%$ of RION, $25 \%$ of CRION, $10 \%$ of SION, $0 \%$ of MSON and $0 \%$ of NMO. As a result patients have been reclassified incorporating their autoantibody status. Presenting visual acuity was significantly worse in patients who were AQP4-lgG seropositive only on CBA ( $p=0.034)$, but there was no relationship between antibody seropositivity and either ON relapse rate or visual acuity outcome.

Conclusions: The number of patients with seronegative CRION and RION has decreased due to improved detection of autoantibodies over the past decade. It remains essential that the clinical phenotype guides both antibody testing and clinical management. Careful monitoring of the disease course is key when considering whether to treat with prophylactic immune suppression.

Key words: optic neuritis, CRION, NMO, aquaporin-4 (AQP4) antibody, myelin oligodendrocyte associated glycoprotein (MOG) antibody 
Page 3 
Page 4

\section{Introduction}

In 2010 we reported the prevalence of NMO-IgG1 in a cohort of patients with optic neuropathies in this journal [1]. NMO-IgG has subsequently been found to be identical to aquaporin-4 IgG (AQP4$\lg G$ ). Subsequent developments have increased the sensitivity and specificity of the AQP4-IgG test [2][2; 3] . In addition, a relevant[4] auto-antibody was re-discovered which targets myelinoligodendrocyte glycoprotein (MOG) [5; 6]tmp[6] . Previously, we identified a subset of relapsing optic neuritis in which discrete acute attacks occur (recurrent isolated optic neuritis, RION); these cases resemble MS or NMO but without supporting evidence for either. This phenotype differs from the previously described chronic relapsing inflammatory optic neuropathy (CRION) [7] characterised by corticosteroid dependence or tendency to early relapse upon withdrawal of corticosteroids. In addition, there is emerging cross-sectional data that patients with both RION and CRION may be seropositive for MOG antibodies [8-10][8-11]tmp . The clinical phenotype of MOG-ON is heterogeneous [12-14]. The need for immunosuppression in patients with CRION strongly suggested an autoimmune pathology [15].

In this study we revisited our original cohort [1] around 10 years later in order to investigate how the clinical diagnosis changed over time. Samples with remaining serum (103/114) were reanalysed for AQP4-IgG and MOG-IgG1 with state of the art live cell based assays (CBA) [6].

\section{Patients and Methods}

The clinical notes of 114 patients presenting with unilateral or bilateral isolated ON who had originally been recruited between November 1995 and September 2007 [1] were reviewed in 2017. The study was approved by the local and national ethics committees. Snellen visual acuities (VA) were converted to digital notation as described [16]. Patients were classified exactly as in our original report [1] according to clinical criteria and response to treatment into one of five 
inflammatory optic neuropathies: multiple sclerosis associated optic neuritis (MSON), singleepisode isolated ON (SION), recurrent isolated ON (RION) CRION or NMO-ON [17] .

\section{Blood samples}

Aliquots of all serum samples were stored at $-80^{\circ} \mathrm{C}$. The samples were coded and send to the respective laboratories for analysis. Serum samples had been tested originally for NMO-

IgG1antibodies at the Mayo Clinic laboratories [2]. In 2015 they were re-tested for MOG-IgG1 at the Oxford Autoimmune Neurology Diagnostic Laboratory as described [9].

Serum was available from 103/114 (91.4\%) of the original samples, including all the AQP4 seropositive samples; the remaining samples were consumed in previous studies. We have indicated In the table how many samples were available for each test at each time point and for each clinical subgroup. There were 30 missing samples for testing for MOG-IgG1.

Cerebrospinal fluid (CSF) oligoclonal bands were determined using 2-4 $\mu \mathrm{L}$ of sample for isoelectric focusing (IEF) on agarose gels and quality control in the London laboratory as described [18].

Serum samples were also used to quantify protein biomarkers which have been related to astrocytic damage in NMO [19; 20] , glial fibrillary acidic protein (GFAP) and S100B using in-house developed ELISAs [21; 22] . Finally, neurofilament protein, a biomarker for neurodegeneration [23] , was quantified by in-house ELISA [24] .

\section{Data analysis}

Data analysis was performed using SAS (V9.4). Data distribution was non-Gaussian and therefore the median and interquartile ranges are shown. Categorical data were compared using the Chisquare test; continuous data using the Kruskal-Wallis test. Correlation analysis was performed using Spearman's R. A p-value of 0.05 was accepted as significant. All tests were two-sided. 


\section{Results}

In total 10 patients developed clinical definite multiple sclerosis, of whom 5 had originally presented as MSON, 2 as SION and 3 as RION. One patient with RION developed CRION. One patient with SION developed a progressive optic neuropathy (PON). One patient with SION (seronegative for AQP4-IgG and MOG-IgG), was later found to have a small perioptic nerve sheath meningioma which could not be seen on the initial imaging study. The single patient with SION who had developed NMO was seronegative for AQP4-IgG and MOG-IgG on all tests. There had been no change of the clinical diagnosis for patients with CRION and NMO. The patient characteristics with antibody data from 2007 and 2017 are shown in Table 1.

No serum sample was positive for both AQP4-IgG and MOG-lgG1. There were no false positive NMO-IgG results in the 2007 data.

AQP4-IgG was detected in six patients previously negative for NMO-IgG1 including two MSON, three CRION and one NMO. The clinical phenotype of these patients remained unchanged, the diagnostic implications of the new autoantibody results are discussed in the next paragraph. MOGIgG was absent in MSON and NMO. MOG-IgG1 was found in $9 \%$ of SION, $20 \%$ of RION and $27 \%$ of CRION.

\section{New auto-antibody related classification}

Table 2 summarises the updated classification of our patient cohort which incorporates AQP4-lgG and MOG-IgG1 seropositivity. Two cases from the previous classification were excluded, one of whom had a perioptic nerve sheath meningioma and the second a progressive rather than relapsing optic neuropathy. By definition in the new group, MOG-ON only consists of patients $(n=11)$ who were tested seropositive for MOG-IgG1. The NMO-ON group increased in numbers from 9 to 19. All other groups were reduced in numbers, MSON by 2 , SION by 6 , RION by 5 and 
CRION by 7. 12 RION and 12 CRION patients remain without known auto-antibody status (missing samples for MOG-IgG in 8, see Table 1).

There is a female predominance in all groups, greatest for RION (75\%) and MOG-ON (73\%), lowest for NMO (63\%). There was a trend for MSON to be the youngest (33 years) and the SION and CRION to be the eldest (44 years). The age difference between groups did however not statistically significance due to small numbers and the large IQR range $(p=0.18)$.

VA at onset $(p=0.08)$ or at follow-up $(p=0.19)$ did not differ between groups. Finally, the number of ON relapses did not differ between RION, CRION, MSON, MOG-ON and NMO-ON ( $p=0.20)$. In the group of MOG-ON patients there was no significant correlation between the MOG titre and either demographic variables or baseline VA $(p=0.77)$, follow-up VA $(p=0.58)$ or number of $O N$ relapses $(p=0.34)$. Likewise, there was no statistical association for the dichotomised data, presence/absence of MOG-IgG1, and number of relapses, visual acuity or visual function outcome. The CSF investigations showed a low percentage of type 2 OCB (= true evidence for intrathecal $\lg G$ synthesis) (Table 3). More frequently there was presence of CSF IgG either in combination with evidence for systemic inflammation or as a monoclonal response $(50 \%$ in RION and $45 \%$ in NMOON, table 3).

There was no significant difference in serum NfH, GFAP or S100B levels between the groups (Table 3). The highest individual serum GFAP levels were however seen in patients with NMO. Interestingly one patient who was classified clinically as NMO, but was seronegative both for NMOlgG1 (2007)and also AQP4-IgG (2017) had serum GFAP level of $2.71 \mathrm{ng} / \mathrm{mL}$ at baseline (October 2004); she subsequently developed a longitudinally extensive transverse myelitis in 2015 at which time she also tested seropositive for AQP4-IgG on a new sample. Serum AQP4-lgG was of prognostic value because those patients who were tested AQP4-IgG positive had significantly worse VA at presentation (VA $0.07 \pm 0.16$ ) when compared to those who were AQP4-IgG seronegative (VA $0.19 \pm 0.29, p=0.034$ ). This level of statistical significance was not observed at the time of our previous report for the NMO-IgG1 test $(p=0.06)$. There was no association of either test 
with the outcome VA or the number of relapses.

\section{Discussion}

The long term follow-up (up to 33 years) $n$ patients presenting with different forms of optic neuritis [1] showed an increase in the autoantibody seropositivity rate from $9 / 114(8 \%)$ to $26(23 \%)$. Seropositivity in CRION increased from $1 / 19(5 \%)$ to $7 / 18$ (38\%). These data are consistent with recent publications showing MOG-IgG1 in patients with CRION [4; 9; 10]. Our current report has the advantage of studying the original CRION cohort. We have been able to demonstrate changes in clinical status and serostatus over time with prolonged clinical follow-up and with improvement in sensitivity, spectrum and specificity of serologic diagnosis (Figure 1). Whilst the clinical phenotype of CRION remained in all patients, one of the CRION patients who was MOG-IgG1 seropositive had developed seizures. This is relevant because the association of MOG with seizures in optic neuritis has previously been reported [25]. Many more cases have since been recognised and importantly treatment with corticosteroids stopped seizures [25-30] .

These results show clearly that the analytical sensitivity of the laboratory autoantibody tests has improved [1]. Importantly all patients with NMO were seronegative for MOG-IgG1 and the diagnosis of NMO remained unchanged at an averaged 10 year follow-up. The technical improvements include two main features. Firstly, live cell based assays are antigen specific with minimal background staining as only the outer surface of the cells are available for antibody binding. NMOIgG IHC uses tissue sections with nuclear and cytosolic determinants available as well as the complete repertoire of rodent brain antigens. Furthermore, the target of the assay is human recombinant antigen rather than rodent antigen. The newer MOG tests require rigorous, prospective validation $[3 ; 31]$ and similar to what has happened to the testing for oligoclonal bands external quality control schemes are required [18; 32].

Although the analysis is based on evaluation of stored samples, IgG is quite stable for at least 25 
years in appropriately stored samples [33]. Although not directly comparable between the two different assays, all NMO-IgG seropositive samples remained seropositive for AQP4-IgG and 14 samples were seropositive on the newer tests suggesting that storage did not result in substantial loss of antibody reactivity. Whether or not IgG directed at MOG behaves different we are unable to tell from present data.

All patients with a clinical diagnosis of CRION or NMO in our original report had been treated with chronic immunosupression regardless of autoantibody status [15]. The one patient with RION who converted to CRION was seronegative both for AQP4-IgG and MOG-IgG. Owing to the presence of significant numbers of individuals with these syndromes who are seronegative for both AQP4-lgG and MOG-IgG and lack other features of MS, our present clinical practice remains guided by the clinical phenotype in seronegative individuals. More data are needed to better understand the heterogeneous clinical presentations associated with MOG seropositivity [8; 9] .

The heterogeneous clinical spectrum of MOG-ON is further highlighted by the observation of a wide range of VA data, both in the literature and in our cohort [9-11; 34-41]. VA outcome may be generally more favourable in patients with MOG-ON compared to those with NMO-ON and CRION, but this observation from the literature could not be confirmed in this cohort because of small patient numbers and high degree of variability. Furthermore, additional factors may need to be considered in interpreting visual outcomes, there is accumulating evidence for substantial inner retinal layer atrophy in MOG-ON patients [42; 43]. Large, collaborative, prospective cohort studies are required to appropriately address the prognostic implications of auto-antibody testing. It will be particularly important to distinguish patients with a mono-phasic disease course from those with a relapsing disease course. The latter group will require particularly careful management as there is a real risk of the cumulative damage of recurrent ON leading to blindness. In addition, MS targeted treatment might cause harm in patients with NMO-ON, CRION or MOG-ON [9; 15; 39]. A progressive optic neuropathy was eventually diagnosed in two cases who were both seronegative for AQP4- and MOG-IgG. In one case this was due to a perioptic meningioma which 
was not visible on the initial dedicated orbital MRI sequences. In the other patient the disease course resembled a hereditary mitochondrial optic neuropathy, although neither genetic testing nor a muscle biopsy confirmed this. Nonetheless, when the course becomes progressive rather than relapsing, this raises concerns about either compression due to neoplasm or a neurodegenerative mechanism.

Compared to the recent literature, the seropositivity rate for MOG-IgG in patients with CRION in our cohort (25\%) is less than the $67-100 \%$ seropositivity rate reported by others $[4 ; 11 ; 44][4]$. Differences in study cohorts may be responsible; one was primarily paediatric (104 children, 7 adults) [4]. In a South Korean cohort, 11/12 (92\%) of patients initially classified as CRION were seropositive for MOG-IgG [11] and perineural enhancement of the optic nerve in 11/17 patients with MOG-ON [11]. We have observed similar findings in new MOG-ON cases. Likewise the Chinese CRION cohort shares similarities with present cohort regarding the poor visual outcome and MRI features, but has an equal gender balance, rather then the female predominance apparent in our CRION cohort [44]. Future studies should address the diagnostic specificity and sensitivity of this radiologic finding [11] in order to guide development of future diagnostic criteria.

One limitation of this study was the inability to retest 11 patients for AQP4-IgG as there was insufficient serum. MOG-IgG1 could not be assessed in 30 patients, 18 of these had MSON, but this could be a contributing factor to the lower 25\% MOG-ON prevalence rate. Likewise we cannot exclude that MOG-IgG is less stable than AQP4-IgG at -80C storage. Another limitation was that recruitment pre-dated the availability of OCT in clinical practice and we were not able to investigate previously observed associations of the antibody status and patterns of retinal layer swelling and atrophy [16]. An important shortcoming of present study is that MRI sequences used when we started to recruit patients with CRION over two decades ago did not permit for imaging at the same level of detail as contemporary sequences. Having recognised these early difficulties with orbital MRI the Queen Square team has contributed to improving the situation by implementation of dedicated sequences. One of the reasons in doing so was the original description of a distinct optic 
nerve lesion pattern in NMO-ON compared to MSON [45]. Consequently, international consensus recommendations have been made regarding a dedicated MRI protocol for the investigation of optic neuritis [16]. Future studies should make use of such a consensus investigation protocol in order to further elucidate the specificity of MRI patterns in different forms of ON.

In conclusion, the clinical phenotype of optic neuritis should continue to guide the clinical

management. In patients with relapsing optic neuritis presence of AQP4-IgG and MOG-lgG renders a diagnosis of MS unlikely, and suggests that immunosuppression should be considered instead of other MS disease modifying treatments, although this recommendation remains based on expert opinion and not high levels of scientific evidence. There is a need for further research to identify the pathophysiology of CRION as many patients with presumed autoimmune pathology remain seronegative for currently known autoimmune targets. There is also a need for future multicentre treatment trials in patients with CRION, NMO-ON and MOG-ON which, because there are no published randomized clinical trials to inform treatment, and all are rare diseases requiring a collaborative network approach.

\section{Acknowledgement}

This research was supported by the National Institute for Health Research (NIHR) Biomedical Research Centre based at Moorfields Eye Hospital NHS Foundation Trust and UCL Institute of Ophthalmology. The views expressed are those of the author(s) and not necessarily those of the NHS, the NIHR or the Department of Health. We apologise to those authors whom we were not able to cite because of space limitations.

\section{Conflict of interest and source of funding}

A Petzold, M Woodhall, Z Khaleeli and GT Plant have no conflict of interest and nothing to disclose. This study was not funded.

P Waters, A Vincent and the University of Oxford hold patents for antibody assays and have received royalties. $P$ Waters has received honoraria from Biogen Idec, Mereo Biopharma, 
Retrogenix, UBC and Euroimmun AG; travel grants from the Guthy-Jackson Charitable Foundation; and research funding from Euroimmun AG.

B Weinshenker receives royalties from RSR Ltd, Oxford University, Hospices Civil de Lyon, and MVZ Labor PD Dr. Volkmann und Kollegen GbR for a patent of NMO-IgG as a diagnostic test for NMO and related disorders. He serves as a member of an adjudication committee for clinical trials in NMO being conducted by Medlmmune and Alexion pharmaceutical companies. He was a consultant for Caladrius Biosciences, Brainstorm Therapeutics, Roivant Sciences and Chugai Pharma regarding potential clinical trials for NMO. He serves as a member of a data safety monitoring committee for clinical trials conducted by Novartis.

S. Pittock has intellectual property associated with the discovery of NMO-IgG, which has been licensed to a commercial entity. The NMO-IgG test is offered on a service basis by Mayo Collaborative Service Inc., an agency of Mayo Foundation. S. Pittock is a named inventor on patents (12/678,350 filed 2010 and 12/573,942 filed 2008) that relate to functional AQP4/NMO-IgG assays and NMO-IgG as a cancer marker; and receives research support from Alexion Pharmaceuticals, Inc., Medimmune LLC and Grifols. He has provided consultation to Alexion Pharmaceutical, Medlmmune LLC, and Chugai Pharma, but has received no personal fees or compensation for these consulting activities. All compensation for consulting activities is paid directly to Mayo Clinic.

\section{Contributionship Statement}

Study design: GTP, AP.

Laboratory work: MW, PW, AV, SP, OT, AP.

Data collection: ZK, AP, MW, OT.

Data analyses and interpretation: AP, GTP, PW, AV, BW, SP, OT, GTP.

Manuscript writing: AP

Manuscript revision: all co-authors. 
Table 1: The patient cohort according to 2007, clinical and MRI based. The patient characteristics are expressed as medians (interquartile range), numbers (\%).

\begin{tabular}{|c|c|c|c|c|c|}
\hline Feature & MSON & SION & RION & CRION & NMO \\
\hline $\mathrm{N}$ & 28 & 41 & 17 & 19 & 9 \\
\hline Age at onset (years) & $33(23-50)$ & $42(15-71)$ & $37(20-69)$ & $45(29-69)$ & $29(25-69)$ \\
\hline Female : Male & $17: 11$ & $26: 15$ & $13: 4$ & $14: 5$ & $6: 3$ \\
\hline NMO-lgG1(2007) & $0 / 28(0 \%)$ & $2 / 41(5 \%)$ & $1 / 17(6 \%)^{1}$ & $1 / 19(5 \%)$ & $5 / 9(56 \%)$ \\
\hline AQP4-IgG (2017) & $2 / 27(7 \%)$ & $2 / 34(6 \%)$ & $1 / 16(7 \%)^{1}$ & $4 / 18(22 \%)$ & $6 / 8(75 \%)$ \\
\hline MOG -lgG1 (2017) & $0 / 9(0 \%)$ & $4 / 40(10 \%)$ & $4 / 16(25 \%)$ & $3 / 12(25 \%)$ & $0 / 7(0 \%)$ \\
\hline Follow-up (years) & $8(7-17)$ & $8(4-10)$ & $12(9-22)$ & $11(8-12)$ & $11(9-12)$ \\
\hline \multirow[t]{6}{*}{ Revised 2017 diagnosis } & 4 RRMS & $1 \mathrm{RRMS}$ & 2 RRMS & & \\
\hline & $1 \mathrm{SPMS}^{2}$ & 1 SPMS & 1 SMPS & & \\
\hline & & $1 \mathrm{PON}$ & $1 \mathrm{CRION}$ & & \\
\hline & & $1 \mathrm{NMO}$ & & & \\
\hline & & 1 perioptic & & & \\
\hline & & meningioma & & & \\
\hline
\end{tabular}

1 These patients have to be re-classified according to the auto-antibody status (see Table 2)

2 This indicates the expected development from a Clinical Isolated Syndrome (CIS) MSON to clinical definite forms of MS, rather than a true changes of diagnosis. 
Page 14

Table 2: The 2017 classification of our patient cohort. The new classification incorporates the autoantibody status in addition to the clinical phenotype, long term clinical follow-up data and MRI. The patient characteristics are expressed as medians (interquartile range), numbers (\%).

\begin{tabular}{lllllll}
\hline \multicolumn{1}{c}{ Feature } & MSON & SION & RION & CRION & NMO-ON & MOG-ON \\
\hline $\mathrm{N}$ & 26 & 35 & 12 & 12 & 19 & 11 \\
\hline Follow-up (years) & $16(5-25)$ & $7(3-11)$ & $10(8-10)$ & $10(8-12)$ & $9(8-12)$ & $16(5-25)$ \\
\hline Age at onset (years) & $33(30-42)$ & $44(32-50)$ & $39(27-45)$ & $44(36-52)$ & $39(26-48)$ & $37(30-55)$ \\
\hline Female : Male & $17: 9$ & $23: 12$ & $9: 3$ & $8: 4$ & $12: 7$ & $8: 3$ \\
\hline VA onset & 0.10 & 0.10 & 0.05 & 0.01 & 0.01 & 0.03 \\
& $(0.03-0.29)$ & $(0.01-0.25)$ & $(0-0.10)$ & $(0-0.17)$ & $(0-0.01)$ & $(0-0.17)$ \\
\hline VA follow-up & 0.67 & 0.38 & 0.25 & 0.17 & 0.17 & 0.58 \\
& $(0.33-1.00)$ & $(0.10-1.00)$ & $(0.10-0.67)$ & $(0.02-0.50)$ & $(0-0.67)$ & $(0.10-0.67)$ \\
\hline ON relapses & $3(2.5-5)$ & $0(0-0)$ & $4.5(4-7)$ & $2(2-4.5)$ & $1(0-4)$ & $1(0-6)$ \\
\hline
\end{tabular}


Table 3: Paraclinical tests in the 2017 classification of our patient cohort.

\begin{tabular}{lllllll}
\hline \multicolumn{1}{c}{ Feature } & MSON & SION & RION & CRION & NMO-ON & MOG-ON \\
\hline Time to sample (days) & 0 & 275 & 1069 & 78 & 842 & 1069 \\
& $(0-4)$ & $(0-933)$ & $(0-1810)$ & $(0-803)$ & $(0-1810)$ & $(0-2072)$ \\
\hline CSF OCB type 2 & $1 / 5(20 \%)$ & $3 / 9(33 \%)$ & 0 & 0 & $2 / 11(18 \%)$ & 0 \\
\hline CSF OCB other & $2 / 5(40 \%)$ & $3 / 9(33 \%)$ & $2 / 4(50 \%)$ & $2 / 6(33 \%)$ & $5 / 11(45 \%)$ & $1 / 7(14 \%)$ \\
\hline Serum NfH [ng/mL] & 0.07 & 0.17 & 0.11 & 0.09 & 0.07 & 0.06 \\
& $(0.04-0.16)$ & $(0.04-0.24)$ & $(0-04-0.18)$ & $(0.01-0.27)$ & $(0.03-0.16)$ & $(0.04-0.16)$ \\
\hline Serum GFAP [ng/mL] & 0 & 0.75 & 0 & 0.63 & 0.67 & 0.34 \\
& & $(0.03-1.47)$ & & $(0.09-0.88)$ & $(0.24-2.24)$ & $(0-0.67)$ \\
\hline Serum S100B [ng/mL] & 0 & 0.06 & 0 & 0.02 & 0 & 0.01 \\
& & $(0.03-0.08)$ & & $(0.01-0.05)$ & $(0-0.08)$ & $(0-0.01)$ \\
\hline AQP4-IgG (2017) & 0 & 0 & 0 & 0 & $15 / 175(79 \%)$ & 0 \\
\hline MOG -IgG1 (2017) & 0 & 0 & 0 & 0 & 0 & $11(100 \%)$ \\
\hline
\end{tabular}

3 The OCB type 2 pattern indicates intrathecal lgG synthesis and has been incorporated in the 2017 revision of the McDonald criteria for MS. Typically the type 2 pattern is referred to as "oligoclonal bands" being present. The percentages (\%) shown were calculated from the total number of CSF samples with matched serum samples.

4 There is a whole range of other diseases where $\operatorname{lgG}$ can be demonstrated in the CSF either monoclonal or oligoclonal with some bands also being present in the matched serum sample [18] .

5 Sample not available in two cases. 
Figure 1: Breakdown of the original CRION cohort over time into specific auto-antibody mediated sub-groups. This flow chart illustrates how a well characterised clinical phenotype of a rare disease permits for prospective interogation of new molecular targets of an autoimmune attack.

\section{References}

[1] Petzold, A.; Pittock, S.; Lennon, V.; Maggiore, C.; Weinshenker, B. G. and Plant, G. T. (2010). Neuromyelitis opticaIgG (aquaporin-4) autoantibodies in immune mediated optic neuritis., J Neurol Neurosurg Psychiatry 81: 109-111.

[2] Waters, P. J.; McKeon, A.; Leite, M. I.; Rajasekharan, S.; Lennon, V. A.; Villalobos, A.; Palace, J.; Mandrekar, J. N.; Vincent, A.; Bar-Or, A. and Pittock, S. J. (2012). Serologic diagnosis of NMO: A multicenter comparison of aquaporin-4lgG assays., Neurology $78: 665-671$.

[3] Waters, P.; Reindl, M.; Saiz, A.; Schanda, K.; Tuller, F.; Kral, V.; Nytrova, P.; Sobek, O.; Nielsen, H. H.; Barington, T.; Lillevang, S. T.; Illes, Z.; Rentzsch, K.; Berthele, A.; Berki, T.; Granieri, L.; Bertolotto, A.; Giometto, B.; Zuliani, L.; Hamann, D.; van Pelt, E. D.; Hintzen, R.; Höftberger, R.; Costa, C.; Comabella, M.; Montalban, X.; Tintoré, M.; Siva, A.; Altintas, A.; Deniz, G.; Woodhall, M.; Palace, J.; Paul, F.; Hartung, H.-P.; Aktas, O.; Jarius, S.; Wildemann, B.; Vedeler, C.; Ruiz, A.; Leite, M. I.; Trillenberg, P.; Probst, M.; Saschenbrecker, S.; Vincent, A. and Marignier, R. (2016).

Multicentre comparison of a diagnostic assay: aquaporin-4 antibodies in neuromyelitis optica., Journal of neurology, neurosurgery, and psychiatry $87: 1005-1015$.

[4] Mayer, M. C.; Breithaupt, C.; Reindl, M.; Schanda, K.; Rostasy, K.; Berger, T.; Dale, R. C.; Brilot, F.; Olsson, T.; Jenne, D.; Probstel, A.-K.; Dornmair, K.; Wekerle, H.; Hohlfeld, R.; Banwell, B.; Bar-Or, A. and Meinl, E. (2013). Distinction and temporal stability of conformational epitopes on myelin oligodendrocyte glycoprotein recognized by patients with different inflammatory central nervous system diseases., The Journal of Immunology 191 : 3594-3604.

[5] Linington, C. and Lassmann, H. (1987). Antibody responses in chronic relapsing experimental allergic encephalomyelitis: correlation of serum demyelinating activity with antibody titre to the myelin/oligodendrocyte glycoprotein (MOG)., Journal of neuroimmunology $17: 61-69$.

[6] Waters, P.; Woodhall, M.; O'Connor, K. C.; Reindl, M.; Lang, B.; Sato, D. K.; Juryńczyk, M.; Tackley, G.; Rocha, J.; Takahashi, T. and others (2015). MOG cell-based assay detects non-MS patients with inflammatory neurologic disease, Neurology-Neuroimmunology Neuroinflammation 2 : e89.

[7] Kidd, D.; Burton, B.; Plant, G. and Graham, E. (2003). Chronic relapsing inflammatory optic neuropathy (CRION), Brain $126: 276-284$.

[8] Petzold, A.; Wong, S. and Plant, G. T. (2016). Autoimmunity in visual loss., Handb Clin Neurol 133 : 353-376.

[9] Chen, J. J.; Flanagan, E. P.; Jitprapaikulsan, J.; López-Chiriboga, A. (S. S.; Fryer, J. P.; Leavitt, J. A.; Weinshenker, B. G.; McKeon, A.; Tillema, J.-M.; Lennon, V. A.; Tobin, W. O.; Keegan, B. M.; Lucchinetti, C. F.; Kantarci, O. H.; McClelland, C. M.; Lee, M. S.; Bennett, J. L.; Pelak, V. S.; Chen, Y.; VanStavern, G.; Adesina, O.-O. O.; Eggenberger, E. R.; Acierno, M. D.; Wingerchuk, D. M.; Brazis, P. W.; Sagen, J. and Pittock, S. J. (2018). Myelin Oligodendrocyte Glycoprotein Antibody (MOG-IgG)-Positive Optic Neuritis: Clinical Characteristics, Radiologic Clues and Outcome., American journal of ophthalmology $195:$ 8-15.

[10] Jitprapaikulsan, J.; Chen, J. J.; Flanagan, E. P.; Tobin, W. O.; Fryer, J. P.; Weinshenker, B. G.; McKeon, A.; 
Lennon, V. A.; Leavitt, J. A.; Tillema, J.-M.; Lucchinetti, C.; Keegan, B. M.; Kantarci, O.; Khanna, C.; Jenkins, S. M.; Spears, G. M.; Sagan, J. and Pittock, S. J. (2018). Aquaporin-4 and Myelin Oligodendrocyte Glycoprotein Autoantibody Status Predict Outcome of Recurrent Optic Neuritis, Ophthalmology 125 : 1628-1637.

[11] Lee, H.-J.; Kim, B.; Waters, P.; Woodhall, M.; Irani, S.; Ahn, S.; Kim, S.-J. and Kim, S.-M. (2018). Chronic relapsing inflammatory optic neuropathy (CRION): a manifestation of myelin oligodendrocyte glycoprotein antibodies., Journal of neuroinflammation $15: 302$.

[12] Jurynczyk, M.; Messina, S.; Woodhall, M. R.; Raza, N.; Everett, R.; Roca-Fernandez, A.; Tackley, G.; Hamid, S.; Sheard, A.; Reynolds, G.; Chandratre, S.; Hemingway, C.; Jacob, A.; Vincent, A.; Leite, M. I.; Waters, P. and Palace, J. (2017). Clinical presentation and prognosis in MOG-antibody disease: a UK study., Brain : a journal of neurology 140 : 3128-3138.

[13] López-Chiriboga, A. S.; Majed, M.; Fryer, J.; Dubey, D.; McKeon, A.; Flanagan, E. P.; Jitprapaikulsan, J.; Kothapalli, N.; Tillema, J.-M.; Chen, J.; Weinshenker, B.; Wingerchuk, D.; Sagen, J.; Gadoth, A.; Lennon, V. A.; Keegan, B. M.; Lucchinetti, C. and Pittock, S. J. (2018). Association of MOG-IgG Serostatus With Relapse After Acute Disseminated Encephalomyelitis and Proposed Diagnostic Criteria for MOG-IgG-Associated Disorders., JAMA neurology $75: 1355-1363$.

[14] Ramanathan, S.; Mohammad, S.; Tantsis, E.; Nguyen, T. K.; Merheb, V.; Fung, V. S. C.; White, O. B.; Broadley, S.; Lechner-Scott, J.; Vucic, S.; Henderson, A. P. D.; Barnett, M. H.; Reddel, S. W.; Brilot, F.; Dale, R. C.; Australasian and Group, N. Z. M. S. (2018). Clinical course, therapeutic responses and outcomes in relapsing MOG antibodyassociated demyelination., Journal of neurology, neurosurgery, and psychiatry $89: 127-137$.

[15] Petzold, A. and Plant, G. T. (2014). Chronic relapsing inflammatory optic neuropathy: a systematic review of 122 cases reported., J Neurol 261 : 17-26.

[16] Petzold, A.; Wattjes, M. P.; Costello, F.; Flores-Rivera, J.; Fraser, C. L.; Fujihara, K.; Leavitt, J.; Marignier, R.; Paul, F.; Schippling, S.; Sindic, C.; Villoslada, P.; Weinshenker, B. and Plant, G. T. (2014). The investigation of acute optic neuritis: a review and proposed protocol., Nature Reviews Neurology 10 : 447-458.

[17] Wingerchuk, D. M.; Lennon, V. A.; Pittock, S. J.; Lucchinetti, C. F. and Weinshenker, B. G. (2006). Revised diagnostic criteria for neuromyelitis optica., Neurology $66: 1485-9$.

[18] Petzold, A. (2013). Intrathecal oligoclonal lgG synthesis in multiple sclerosis., Journal of Neuroimmunology 262 : 1 10.

[19] Misu, T.; Takano, R.; Fujihara, K.; Takahashi, T.; Sato, S. and Itoyama, Y. (2009). Marked increase in cerebrospinal fluid glial fibrillar acidic protein in neuromyelitis optica: an astrocytic damage marker., J Neurol Neurosurg Psychiatry $80: 575-577$.

[20] Petzold, A.; Marignier, R.; Verbeek, M. M. and Confavreux, C. (2011). Glial but not axonal protein biomarkers as a new supportive diagnostic criteria for Devic neuromyelitis optica? Preliminary results on 188 patients with different neurological diseases., J Neurol Neurosurg Psychiatry 82 : 467-469.

[21] Petzold, A.; Keir, G.; Lim, D.; Smith, M. and Thompson, E. (2003). CSF and serum S100B: release and wash-out pattern, Brain Res Bull $61: 281-285$.

[22] Petzold, A.; Keir, G.; Green, A. J. E.; Giovannoni, G. and Thompson, E. J. (2004). An ELISA for glial fibrillary acidic protein., J Immunol Methods 287 : 169-177.

[23] Khalil, M.; Teunissen, C. E.; Otto, M.; Piehl, F.; Sormani, M. P.; Gattringer, T.; Barro, C.; Kappos, L.; Comabella, M.; Fazekas, F.; Petzold, A.; Blennow, K.; Zetterberg, H. and Kuhle, J. (2018). Neurofilaments as biomarkers in neurological disorders., Nature Reviews Neurology 14 : 577-589. 
[24] Petzold, A.; Keir, G.; Green, A.; Giovannoni, G. and Thompson, E. (2003). A specific ELISA for measuring neurofilament heavy chain phosphoforms, J Immunol Methods 278 : 179-190.

[25] Gutman, J. M.; Kupersmith, M.; Galetta, S. and Kister, I. (2018). Anti-myelin oligodendrocyte glycoprotein (MOG) antibodies in patients with optic neuritis and seizures., Journal of the neurological sciences $387: 170-173$.

[26] Ogawa, R.; Nakashima, I.; Takahashi, T.; Kaneko, K.; Akaishi, T.; Takai, Y.; Sato, D. K.; Nishiyama, S.; Misu, T.; Kuroda, H.; Aoki, M. and Fujihara, K. (2017). MOG antibody-positive, benign, unilateral, cerebral cortical encephalitis with epilepsy., Neurology $(\mathrm{R})$ neuroimmunology \& neuroinflammation 4 : e322.

[27] Adachi, H.; Ide, Y.; Takahashi, T.; Yoneda, Y. and Kageyama, Y. (2018). [Cerebral cortical encephalitis with antimyelin oligodendrocyte glycoprotein (MOG) antibody]., Rinsho shinkeigaku = Clinical neurology $58:$ 767-770.

[28] Ramanathan, S.; O'grady, G. L.; Malone, S.; Spooner, C. G.; Brown, D. A.; Gill, D.; Brilot, F. and Dale, R. C. (2018). Isolated seizures during the first episode of relapsing myelin oligodendrocyte glycoprotein antibody-associated demyelination in children., Developmental medicine and child neurology .

[29] Hamid, S. H. M.; Whittam, D.; Saviour, M.; Alorainy, A.; Mutch, K.; Linaker, S.; Solomon, T.; Bhojak, M.; Woodhall, M.; Waters, P.; Appleton, R.; Duddy, M. and Jacob, A. (2018). Seizures and Encephalitis in Myelin Oligodendrocyte Glycoprotein IgG Disease vs Aquaporin 4 lgG Disease., JAMA neurology 75 : 65-71.

[30] Yao, Y.; Xu, Y.; Ren, H.; Zhou, X.; Jin, L.; Huang, Y.; Lu, Q.; Yang, X.; Zhang, Y.; Zhu, Y.; Peng, B. and Cui, L. (2019). Acute epileptic seizures in myelin oligodendrocyte glycoprotein encephalomyelitis and neuromyelitis optica spectrum disorder: A comparative cohort study., Multiple sclerosis and related disorders 27 : 281-288.

[31] Jarius, S.; Paul, F.; Aktas, O.; Asgari, N.; Dale, R.; de Seze, J.; Franciotta, D.; Fujihara, K.; Jacob, A.; Kim, H. and others (2018). MOG encephalomyelitis: international recommendations on diagnosis and antibody testing, Journal of Neuroinflammation $15: 134$.

[32] Petzold, A. (2018). Applying the 2017 McDonald diagnostic criteria for multiple sclerosis., The Lancet. Neurology 17 : 496-497.

[33] Gislefoss, R. E.; Grimsrud, T. K. and Mørkrid, L. (2009). Stability of selected serum proteins after long-term storage in the Janus Serum Bank., Clinical chemistry and laboratory medicine $47: 596-603$.

[34] Kitley, J.; Waters, P.; Woodhall, M.; Leite, M. I.; Murchison, A.; George, J.; KÃ14ker, W.; Chandratre, S.; Vincent, A. and Palace, J. (2014). Neuromyelitis Optica Spectrum Disorders With Aquaporin-4 and Myelin-Oligodendrocyte Glycoprotein Antibodies: A Comparative Study., JAMA Neurol 71 :276-283.

[35] Ramanathan, S.; Reddel, S. W.; Henderson, A.; Parratt, J. D. E.; Barnett, M.; Gatt, P. N.; Merheb, V.; Kumaran, R.Y. A.; Pathmanandavel, K.; Sinmaz, N.; Ghadiri, M.; Yiannikas, C.; Vucic, S.; Stewart, G.; Bleasel, A. F.; Booth, D.; Fung, V. S. C.; Dale, R. C. and Brilot, F. (2014). Antibodies to myelin oligodendrocyte glycoprotein in bilateral and recurrent optic neuritis., Neurol Neuroimmunol Neuroinflamm 1 : e40.

[36] Martinez-Hernandez, E.; Sepulveda, M.; RostÃ $; s y$, K.; HÃ fftberger, R.; Graus, F.; Harvey, R. J.; Saiz, A. and Dalmau, J. (2015). Antibodies to Aquaporin 4, Myelin-Oligodendrocyte Glycoprotein, and the Glycine Receptor $\hat{I} \pm$ 1 Subunit in Patients With Isolated Optic Neuritis., JAMA Neurol 72 : 187-193.

[37] Matsuda, R.; Kezuka, T.; Umazume, A.; Okunuki, Y.; Goto, H. and Tanaka, K. (2015). Clinical Profile of Anti-Myelin Oligodendrocyte Glycoprotein Antibody Seropositive Cases of Optic Neuritis, Neuro-Ophthalmology 39 : 213-219.

[38] Moura, F. C.; Sato, D. K.; Rimkus, C. M.; Apóstolos-Pereira, S. L.; de Oliveira, L. M.; Leite, C. C.; Fujihara, K.; Monteiro, M. L. R. and Callegaro, D. (2015). Anti-MOG (Myelin Oligodendrocyte Glycoprotein)-Positive Severe Optic Neuritis with Optic Disc Ischaemia and Macular Star, Neuro-Ophthalmology : 1-4. 
[39] Jarius, S.; Ruprecht, K.; Kleiter, I.; Borisow, N.; Asgari, N.; Pitarokoili, K.; Pache, F.; Stich, O.; Beume, L.-A.; Hümmert, M. W.; Ringelstein, M.; Trebst, C.; Winkelmann, A.; Schwarz, A.; Buttmann, M.; Zimmermann, H.; Kuchling, J.; Franciotta, D.; Capobianco, M.; Siebert, E.; Lukas, C.; Korporal-Kuhnke, M.; Haas, J.; Fechner, K.; Brandt, A. U.; Schanda, K.; Aktas, O.; Paul, F.; Reindl, M.; Wildemann, B. and in cooperation with the Neuromyelitis Optica Study Group (NEMOS) (2016). MOG-IgG in NMO and related disorders: a multicenter study of 50 patients. Part 2: Epidemiology, clinical presentation, radiological and laboratory features, treatment responses, and long-term outcome., Journal of neuroinflammation $13: 280$.

[40] Pache, F.; Zimmermann, H.; Mikolajczak, J.; Schumacher, S.; Lacheta, A.; Oertel, F. C.; Bellmann-Strobl, J.; Jarius, S.; Wildemann, B.; Reindl, M.; Waldman, A.; Soelberg, K.; Asgari, N.; Ringelstein, M.; Aktas, O.; Gross, N.; Buttmann, M.; Ach, T.; Ruprecht, K.; Paul, F.; Brandt, A. U. and in cooperation with the Neuromyelitis Optica Study Group (NEMOS) (2016). MOG-IgG in NMO and related disorders: a multicenter study of 50 patients. Part 4: Afferent visual system damage after optic neuritis in MOG-IgG-seropositive versus AQP4-lgG-seropositive patients., Journal of neuroinflammation $13: 282$.

[41] Havla, J.; Kümpfel, T.; Schinner, R.; Spadaro, M.; Schuh, E.; Meinl, E.; Hohlfeld, R. and Outteryck, O. (2017). Myelin-oligodendrocyte-glycoprotein (MOG) autoantibodies as potential markers of severe optic neuritis and subclinical retinal axonal degeneration., Journal of neurology $264: 139-151$.

[42] Mekhasingharak, N.; Laowanapiban, P.; Siritho, S.; Satukijchai, C.; Prayoonwiwat, N.; Jitprapaikulsan, J.; Chirapapaisan, N. and Group, S. N. R. (2018). Optical coherence tomography in central nervous system demyelinating diseases related optic neuritis., International journal of ophthalmology $11: 1649-1656$.

[43] Narayan, R. N.; McCreary, M.; Conger, D.; Wang, C. and Greenberg, B. M. (2019). Unique characteristics of optical coherence tomography (OCT) results and visual acuity testing in myelin oligodendrocyte glycoprotein (MOG) antibody positive pediatric patients., Multiple sclerosis and related disorders 28 : 86-90.

[44] Liu, H.; Zhou, H.; Wang, J.; Xu, Q. and Wei, S. (2018). Antibodies to myelin oligodendrocyte glycoprotein in chronic relapsing inflammatory optic neuropathy., The British journal of ophthalmology .

[45] Storoni, M.; Davagnanam, I.; Radon, M.; Siddiqui, A. and Plant, G. T. (2013). Distinguishing optic neuritis in neuromyelitis optica spectrum disease from multiple sclerosis: a novel magnetic resonance imaging scoring system., J Neuroophthalmol $33: 123-127$. 